\title{
La educación secundaria y superior en Colombia vista desde las pruebas Saber
}

Mayerlin Castro Ávila

Matemática

Secretaria de Educación de Boyacá - Colombia

mayis-200@hotmail.com

https://orcid.org/0000-0003-0101-9902

\section{Jorge Ruiz Linares}

Doctor en Recursos Naturales

Universidad Pedagógica

Tecnológica de Colombia

jorge.ruiz@uptc.edu.co

https://orcid.org/0000-0003-0376-8608

\section{Artículo de Investigación}

Recepción: 6 de mayo de 2019

Aprobación: 5 de agosto de 2019

https://doi.org/10.19053/22160159.v10.n25.2019.9465

\section{Resumen}

Las pruebas estándar generan debate al evaluar la calidad de la educación. Sin embargo, son una herramienta de caracterización de los estudiantes. Colombia parece ser el único país del mundo en que se puede hacer el seguimiento de la prueba estándar de secundaria y universitaria para el mismo estudiante. El estudio busca caracterizar a los estudiantes que presentaron ambas pruebas. Se llevan a cabo tablas de contingencia en Excel para los estudiantes en Colombia que lograron terminar su bachillerato y pregrado; presentaron la prueba Saber 11 entre 2005 y 2006; y -4 o 5 años después- presentaron la prueba Saber Pro en instituciones técnicas y universitarias y solo universitarias. Seis de cada diez estudiantes que terminaron sus estudios superiores asistieron a un colegio o a una universidad del sector privado. Aproximadamente dos de cada tres estudiantes que terminan sus estudios superiores lo hacen en una universidad. Hay diferencias significativas 
en las pruebas Saber 11 por género, que están correlacionadas con la prueba Saber Pro. Los resultados de las pruebas Saber Pro en universidades oficiales son superiores a los de las privadas.

Palabras clave: calidad de la educación, Colombia, enseñanza secundaria, enseñanza superior, pruebas estándar

\title{
High school and higher education in Colombia seen from the Saber tests perspective
}

\begin{abstract}
Standardized testing generates debate when it comes to assessing the quality of education. However, it constitutes a tool for characterization of students. Colombia seems to be the only country in the world where it is possible to monitor a student's high school and university standardized tests performance. This study aims to characterize the students who took both tests. Contingency tables in Excel were created using data from students in Colombia who finished high school and earned a bachelor's degree; those who took the Saber 11 test between 2005 and 2006 and, 4 or 5 years afterwards, they took the Saber Pro test in institutes of a technical and university nature or just universities. 6 out of 10 students who finished their higher education studies attended private high schools or private universities. Approximately 2 out of 3 students finish their higher education studies at a university. Significant differences by gender exist in the Saber 11 test, which are correlated with the Saber Pro test. Public universities have higher Saber Pro scores than private universities.
\end{abstract}

Keywords: quality of education, Colombia, secondary education, higher education, standardized tests

\section{L'enseignement secondaire et supérieur en Colombie dans la perspective des examens Saber}

\section{Résumé}

Les examens standardisés donnent lieu à des débats sur l'évaluation de la qualité de l'éducation. Toutefois, ils constituent un outil de caractérisation des étudiants. La Colombie semble être le seul pays où il 
est possible de faire le suivi de la performance d'un étudiant aux examens standardisés de l'enseignement secondaire et universitaire. Cette étude cherche à caractériser les étudiants qui ont passé les deux examens. Des tableaux de contingence ont été créés dans Excel à partir des données des étudiants en Colombie qui ont fini leurs études secondaires et de licence ; ceux qui ont passé l'examen Saber 11 entre 2005 et 2006, et 4 ou 5 ans plus tard, ils ont passé l'examen Saber Pro dans des institutes techniques, institutes universitaires, ou simplement dans des universités. Six étudiants ayant fini leurs études sur dix ont fréquenté un lycée ou une université appartenant au secteur privé. Environ deux étudiants sur trois finissent leurs études supérieures dans une université. Il existe des différences significatives entre les sexes dans les examens Saber 11, qui sont corrélées avec l'examen Saber Pro. Les universités publiques ont de meilleurs résultats aux examens Saber que les universités privées.

Mots-clés : qualité de l'éducation, Colombie, enseignement secondaire, enseignement supérieur, évaluation des étudiants

\section{A educação secundária e superior na Colômbia vista desde as provas Saber}

\section{Resumo}

As provas standard geram debate ao avaliar a qualidade da educação. No entanto, são uma ferramenta de caracterização dos estudantes. Colômbia parece ser o único país do mundo em que se pode fazer o rastreamento da prova standard de secundária e universitária para o mesmo estudante. $\mathrm{O}$ estudo procura caracterizar aos estudantes que apresentaram ambas as provas. Levam-se a cabo tabelas de contingencia em Excel para os estudantes na Colômbia que conseguiram terminar seu ensino secundário e nível de graduação; apresentaram a prova Saber 11 entre 2005 e 2006; e -4 ou 5 anos depois - apresentaram a prova Saber Pro em instituições técnicas e universitárias e só universitárias. Seis em cada dez estudantes que terminaram seus estudos superiores assistiram a um colégio ou a uma universidade do sector privado. Aproximadamente dois em cada três estudantes que terminam seus estudos superiores o fazem numa universidade. Há diferenças significativas nas provas Saber 11 por género, que estão correlacionadas com a prova Saber Pro. Os resultados das provas Saber Pro em universidades oficiais são superiores às das privadas.

Palavras-chave: qualidade da educação, Colômbia, ensino secundário, ensino superior, avaliação do estudante 


\section{El debate sobre la calidad de la educación y su evaluación}

En los últimos tiempos han surgido conceptos y categorías que han modificado la manera de percibir los procesos educativos. La sociedad actual plantea a la educación múltiples retos que impulsan a la comunidad académica a investigar y profundizar los conocimientos frente a los fenómenos y determinantes asociados a la calidad dela educación. El concepto de calidad y su evaluación tienen su génesis en las organizaciones mercantilistas y llega al ámbito educativo luego de haberse implantado en la industria (Alonso, Rebolloso, Pozo \& Fernández, 1999). La cobertura y la calidad de la educación se convierten en herramientas de política pública que pueden reducir los niveles de pobreza de un país y aumentar la movilidad social de sus individuos (Barrera-Osorio, Maldonado \& Rodríguez, 2012). En ese orden de ideas y de ser posible, el sistema ideal debería estar organizado de forma que se disipen las desigualdades de género que vienen de los hogares. No es de extrañar entonces que, en la actualidad, y desde hace más de un cuarto de siglo, entre los conceptos y palabras de fuerte carga simbólica figuran, sin duda, los de calidad y evaluación que, no por azar, aparecen con gran frecuencia unidas reforzándose mutuamente (Orden, 2009).

Los conceptos de evaluación y calidad de la educación se caracterizan por tener múltiples percepciones (López, 2007). Según Ardila (2011) el concepto de calidad hace referencia a la capacidad de las instituciones educativas para lograr que sus alumnos alcancen metas educativas independientemente de su origen social; mientras que Unicef (2010) aborda una aproximación comprensiva de calidad en la educación, aquella que involucra aprendices, contenido, procesos, medio ambiente y resultados.

Actualmente hay dos aproximaciones que enmarcan el debate acerca de la calidad en la educación: la de la teoría del capital humano y la de los derechos humanos. A ambas se les ha criticado por quedarse cortas frente a la teoría de la justicia social (Tikly \& Barrett, 2011). A la luz de la globalización, la educación juega un papel medular en el desarrollo de las naciones (Carter \& O'Neill, 1995). En otras palabras, volviendo a la teoría del capital humano, se invierte en educación y en la calidad de la educación por el papel 
que estos tienen en el desarrollo y, más específicamente, por su impacto en el incremento del producto interno bruto. Winters (2011) encontró que en ciudades donde se encontraba una universidad, el capital humano y la calidad de vida aumentaba. En contraste con el énfasis en el crecimiento económico dentro de la aproximación del capital humano, los defensores de la aproximación de los derechos humanos ven el desarrollo como multifacético, ya que envuelve un espectro de dimensiones económicas, políticas y culturales, vinculadas a la paz, seguridad humana y sustentabilidad ambiental (Piron \& O’Neill, 2005). El argumento principal a favor de la aproximación de la justicia social en la educación y su calidad es una nueva forma de pensar, enraizada en las libertades individuales, que a la vez comprenden las aproximaciones del capital humano y de los derechos humanos (Tikly \& Barrett, 2011).

Desde Charniak (1972), se sabe que es necesario contar con gran cantidad de conocimiento para comprender los textos más sencillos. Hay cuatro tipos de saberes: un saber conceptual o simplemente saber, un saber procedimental o saber hacer, un saber actitudinal o saber ser y, finalmente, un saber meta-cognitivo o saber aprender (Pinto, 1999). El común denominador en América Latina es el diseño de currículos que buscan destreza en estos cuatro saberes (Ferrer, Valverde \& Esquivel, 1999). No obstante, sin acceso a una educación de calidad, el anterior enunciado no tiene significado alguno.

De otro lado, Chumacero, Gómez y Paredes (2011) encontraron que en Chile tanto la distancia del hogar a la institución como la calidad de las instituciones educativas explican la selección de estas, lo cual hace cuestionar los resultados de encuestas que presentaban la distancia como la variable más importante al escoger una institución, muy por encima de los resultados de las pruebas normalizadas.

\section{La calidad de la educación y su evaluación en Colombia}

La problemática de la calidad en educación superior en Colombia emerge de la incapacidad por parte de la comunidad educativa e instancias gubernamentales para afrontar los retos que les imponen la evolución en los fenómenos sociales, económicos, culturales, científicos y políticos y su incidencia en el desarrollo educativo 
internacional, nacional, local y regional (Ardila, 2011). Esto solo puede ser superado si existe un diagnóstico de la situación de la educación colombiana. A pesar de los logros en el sector educativo, hay retos como aumentar la cobertura educativa, mejorar la calidad y la pertinencia, y por último, contar con una administración y finanzas apropiadas (OECD \& World Bank, 2013).

La calidad de la educación en Colombia se ha puesto en el centro del debate educativo y ha escalado a las más altas esferas de la política pública del país, al ser un programa bandera del presidente Santos en su segundo mandato. En los últimos años se presentó un aumento considerable en el presupuesto oficial, tanto nacional como departamental de los planteles de educación media (Gaviria \& Barrientos, 2001). Lo anterior, junto con la descentralización de las finanzas, mejoró las tasas de matrícula en colegios oficiales (Faguet \& Sánchez, 2008). Orozco (2008) opina que, sin calidad, el aumento de la cobertura en la matrícula es una causa perdida. Saavedra (2012) va más lejos al subrayar que pocos gobiernos han podido aumentar simultáneamente la cobertura y la calidad en educación. En efecto, el estudio de Camacho, Messina y Uribe (2016) sugiere que la rápida expansión de programas profesionales en Colombia posiblemente deterioró su calidad.

Hay una amplia evidencia que indica que el acceso a las universidades en Colombia presenta enormes disparidades socioeconómicas y por región (Melguizo, Sánchez \& Velasco, 2016) e incluso a nivel municipal (Loaiza \& Hincapié, 2016). Al sector rural se le ha catalogado como el que se encuentra en la trampa de pobreza por la falta de acceso a la educación, entre otros factores (Rodríguez, Sánchez \& Armenta, 2010). El conflicto armado en Colombia es otro ingrediente que compromete la educación (Gómez, 2016) y contribuye a las diferencias educativas urbanas y rurales. Además, hay que tener en cuenta que las decisiones sobre elegir una institución también se ven afectadas por la ubicación y la distancia entre el hogar y la institución (Burgess \& Briggs, 2010). Guhl (2005) concluye que los aspirantes a las sedes de la Universidad Nacional de Colombia se determinan por un radio de $200 \mathrm{~km}$, mientras que Mina (2004) encuentra que la distancia a la ciudad capital está negativamente asociada con los puntajes Ecaes. Asimismo, se encuentra que existen en el país significativas diferencias en los puntajes de las pruebas Saber 11 y Saber Pro obtenidos por 
estudiantes provenientes de planteles públicos y privados, situación que se exacerba cuando se comparan entre la universidad pública y privada (Celis, Jiménez \& Jaramillo, 2012).

El programa Ser Pilo Paga fue lanzado en 2014 y se basó en la prueba Saber 11 para otorgar crédito educativo subsidiado y ofrecer becas de sostenimiento a estudiantes en universidades acreditadas. Este programa parece haber sido efectivo en incrementar el número de estudiantes de bajos ingresos en estas universidades (Melguizo, Sánchez \& Velasco, 2016).

\section{El sistema de evaluación}

Común a todas las evaluaciones es la comparación de los resultados frente a algún referente. Valverde (1997) postula que el currículum prescrito es aquel conjunto de decisiones y objetivos que el sistema educativo aspira a transmitir. Tales decisiones se toman en distintos niveles, desde el nacional hasta el aula misma (Ferrer, Valverde \& Esquivel, 1999). Este es el referente que se desea evaluar con las pruebas. Así, la evaluación normalizada ayuda a los planteles a establecer metas y a los alumnos y maestros a aprender y enseñar mejor (Linn \& Herman, 1997).

Las pruebas que el Icfes realiza para evaluar la educación se pueden dividir en tres grandes grupos: las de educación media Saber 11-, superior - Saber Pro, antes llamada Ecaes-y el Programa para la Evaluación Internacional de Estudiantes [Programme for International Student Assessment-PISA] de la Organización para la Cooperación y el Desarrollo Económicos [OCDE], las cuales comparan desde el 2006 el desempeño colombiano frente al de otros países. En la voz de Ferrer y Arregui (2003), estas últimas ayudan a mejorar la calidad y eficiencia de las pruebas nacionales y, según el Banco Mundial (2009), son significativas en el momento de establecer un parámetro sobre la calidad y equidad del sistema educativo colombiano.

Entre las bondades de aplicar una prueba modelo, como Saber 5, 9, 11 o Saber Pro, está su alta validez externa; es decir, poder medir desempeño educativo en distintos lugares, poder compararlos y establecer conductores y tendencias, entre otros. Son de utilidad a muchos actores, entre los que cabe mencionar el Estado y, en 
particular, el Ministerio de Educación Nacional, las secretarías de educación departamental, los establecimientos y los propios estudiantes, para que se tomen decisiones informadas. Dichas pruebas pueden llegar a afectar la asignación de la cantidad de recursos con base en deficiencias o logros obtenidos. Sin embargo, se ha advertido el peligro de aplicar a la educación las normas del mercado, que al asumirla como un simple bien transable, se le puede reducir al nivel de mercancía (Tünnermann, 2008).

Con una tendencia a la delegación de la responsabilidad del poder central al local en la educación, las pruebas cobran mucha importancia, a nivel nacional, regional y local, ya que, según Montoya (2010), pueden hacer una diferencia en la calidad de la educación sin mayores inversiones. En el nivel local, las pruebas sobre rendimiento académico hacen más transparente al sistema educativo al proveer información sobre la demanda y la oferta, para llegar a una opinión informada (Ferrer \& Arregui, 2003).

Colombia es, al parecer, el único país del mundo donde se puede hacer un seguimiento del mismo estudiante con las pruebas de Estado estandarizadas al terminar la secundaria y la universidad (Castro, Ruiz \& Guzmán, 2017). Se reportan los resultados de todos los estudiantes en Colombia que presentaron la prueba Saber 11 para 2005-2006 y, 4 o 5 años después, la prueba Saber Pro. Es la primera vez que un estudio de esta naturaleza se publica en una revista arbitrada.

\section{Objetivos}

El objetivo del presente estudio es caracterizar a todos los estudiantes en Colombia que presentaron la prueba Saber 11 para 2005-2006 y, 4 o 5 años después, la prueba Saber Pro, así como establecer la asociación de ambas pruebas en relación con diferentes variables como género, edad, carácter de las instituciones y resultados de las pruebas, entre otros.

\section{Materiales y métodos}

Las bases de datos de las pruebas Saber se descargaron en noviembre de 2015 del portal del Icfes ${ }^{1}$, mediante la apertura de una

$\overline{1 \quad \text { www.icfes.gov.co }}$ 
cuenta en la pestaña de investigadores. En ese momento, la prueba Saber Pro solamente estaba disponible para los años 2009, 2010 y 2011. Sin embargo, al revisar las bases de datos se observó que solamente para los años 2009 y 2010 se tenía disponible la variable que corresponde al puntaje de la prueba específica, es decir, la propia de la prueba aplicada para cada una de las áreas del conocimiento, situación contraria a la que se encontró para la base de 2011, que presenta resultados de las pruebas generales -dadas para todos los inscritos en las pruebas-. Por consiguiente, se decidió no descargar la base de datos para el 2011.

Para el caso de la prueba Saber 11, se encontraron 902984 registros de estudiantes para los años 2005 y 2006, mientras que para el caso de la prueba Saber Pro para los años 2009 y $2010-$ cuatro y cinco años después-, se encontraron tan solo 413584 registros de estudiantes. Para ambas pruebas se descargó la información referente al primer y al segundo semestre de cada año. En cuanto a la prueba Saber 11, se estableció un filtro, tomando tan solo aquellos estudiantes que tenían cumplidos los 14 años, pero se dejó sin restricción la edad superior. El trabajo previo de Celis, Jiménez y Jaramillo (2012) también consideró la edad mínima de 14 años, pero a diferencia del presente estudio, dichos autores pusieron como edad máxima los 20 años.

Las bases de datos -Saber 11: 2005 y 2006; Saber Pro: 2009 y 2010 - fueron las seleccionadas para el presente estudio, dado que contienen la información de las dos pruebas específicas, así como otras variables indispensables para el desarrollo de este proyecto. Puesto que esto no sucede con las bases de datos posteriores al año 2010, cuya información se relaciona únicamente con las pruebas genéricas, no se utilizaron dichas bases de datos para este estudio.

Además, con el fin de llevar a cabo el cruce entre las pruebas Saber 11 y Saber Pro, se descargó el archivo que contiene el código del Icfes, un único número identificador que permite vincular la información para cada estudiante que tomó ambas pruebas. Estas dos bases de datos se descargaron por defecto en el programa Access de Microsoft. No obstante, posteriormente se exportaron a Microsoft Excel, por considerarse este programa más conveniente para la manipulación de la información, hacer tabulaciones cruzadas, filtrado de datos y obtención de estadísticas. 
El siguiente paso consistió en identificar las variables comunes a las bases de datos en los diferentes años y semestres, puesto que no todas contienen la misma información. Se eliminaron las variables que no contenían información. Para el caso de los años 2005 y 2006, primer y segundo semestre, las variables comunes a estas cuatro bases de datos de la prueba Saber 11 fueron: año y semestre de presentación, identificación consecutiva única del estudiante que presentó la prueba Saber 11, género del estudiante, fecha de presentación, día de nacimiento, mes de nacimiento, año de nacimiento, código del municipio donde reside, nombre del municipio de residencia, departamento de residencia, código del municipio de presentación de la prueba, nombre del municipio de presentación de la prueba, departamento de presentación de la prueba, código del Servicio Nacional de Pruebas del colegio en que cursó o terminó el bachillerato, nombre del colegio, calendario del colegio, carácter del colegio, jornada del colegio, valor de la pensión, puntaje de biología, puntaje de matemáticas, puntaje de filosofía, puntaje de física, puntaje de química, puntaje de lenguaje, puntaje de sociales y puntaje de idioma.

Para el caso delas cuatro bases de datos dela prueba Saber Pro, para los años 2009 y 2010, primer y segundo semestre, las variables fueron: año y semestre de presentación, identificación consecutiva única del estudiante que presentó la prueba Saber Pro, fecha de presentación, día de nacimiento, mes de nacimiento, año de nacimiento, estado civil, código de la institución, origen de la institución, carácter académico de la institución, programa académico, código DANE del municipio, jornada de la institución, valor de la matrícula, código de educación del padre, código de educación de la madre, código de ocupación del padre, código de ocupación de la madre, estrato, municipio de presentación de la prueba, departamento de presentación de la prueba, puntaje específico.

A continuación, se vincularon las ocho bases de datos de las dos pruebas, Saber 11 y Saber Pro. Cada fila corresponde a un estudiante, donde primero aparecen las variables y los resultados de la prueba Saber 11 seguidos por las variables y resultados de la prueba Saber Pro.

Teniendo la base de datos consolidada con todas las variables, se prosiguió a la realización de nuevos cálculos. Se sintetizaron 
algunas de las variables, de modo que se pudieran realizar más análisis y comparaciones entre las dos pruebas, tal como se describe a continuación.

En primer lugar, se calculó la edad juliana para cada estudiante en el momento de presentar la prueba Saber 11 y Saber Pro. La justificación para el uso de esta edad radica en que en el calendario gregoriano el número de días que tienen los meses presenta variación; 28, 29-cada cuatro años-, 30 o 31 días. Esta situación introduce ruido en programación. Teniendo el día, mes y año de nacimiento de los estudiantes, se procedió a utilizar estos datos para calcular la edad juliana, que está dada por la siguiente fórmula:

Edad juliana $=a \tilde{n} o^{*} 1000+(($ mes-1)*31)-día

Asímismo, secalculó el tiempotranscurrido entrela presentación de ambas pruebas para cada estudiante. Esto se obtuvo de restar la edad juliana al presentar Saber Pro de la edad juliana al presentar Saber 11. Con el fin de verificar la consistencia de la información de ambas pruebas por estudiante, se revisó que la variable edadjuliana calculada en ambas pruebas, coincidiera; en otras palabras, la resta de estas debe ser igual a cero; en caso contrario, se eliminaron estos registros.

A partir de la Resolución $N^{\circ} 489$ de octubre 20 de 2008 del Icfes, se calculó el índice sintético, el cual consolida los ocho puntajes de las pruebas que hacen parte del núcleo común de la prueba Saber 11. Para esto se tomaron los ocho puntajes del núcleo común y se normalizaron para generar promedios. Esto es, al puntaje de un estudiante se le resta el promedio y el resultado se divide por la desviación estándar de ese promedio. Si la calificación del alumno $\alpha$, en la prueba $\rho$ es P $\rho \alpha$ el promedio de los puntajes P $\rho a$ es Prom $(\mathrm{P} \rho)$ y la desviación de ese promedio es $\operatorname{Des}(\mathrm{P} \rho)$; entonces la calificación normalizada del alumno $\alpha$ en la prueba $\rho$ es:

$$
\mathrm{NP}^{\rho \alpha}=\frac{\mathrm{P} \rho \alpha-\operatorname{Prom}(\mathrm{P} \rho)}{\operatorname{Des}(\mathrm{P} \rho)}
$$

Con las calificaciones normalizadas de las pruebas de Química (q), Física (f), Biología (b), Sociales (s), Filosofía (p), Matemáticas (m), Lenguaje (l) e Inglés (i) del estudiante $\alpha$, se calculó el promedio ponderado de sus puntajes normalizados: 
$\mathrm{PPN} \alpha=\frac{\mathrm{NP} q \alpha+\mathrm{NP} f \alpha+\mathrm{NP} b \alpha+2 * \mathrm{NP} s \alpha+\mathrm{NPp} \alpha+3 * \mathrm{NP} m \alpha+3 * \mathrm{NP} l \alpha+\mathrm{NP} i \alpha}{13}$

Y la desviación de estas calificaciones - no ponderadas-:

$D S P \alpha=\sqrt{\frac{\begin{array}{l}{[\mathrm{NP} q \alpha-P S \alpha)^{2}+(\mathrm{NP} f \alpha-P S \alpha)^{2}+(\mathrm{NP} b \alpha-P S \alpha)^{2}+(\mathrm{NP} s \alpha-P S \alpha)^{2}+} \\ \left.(\mathrm{NPp} \alpha-P S \alpha)^{2}+(\mathrm{NP} m \alpha-P S \alpha)^{2}+(\mathrm{NPl} \alpha-P S \alpha)^{2}+(\mathrm{NP} i \alpha-P S \alpha)^{2}\right]\end{array}}{8}}$

Donde PSa es el promedio simple de las calificaciones normalizadas del estudiante $\alpha$, es decir:

$$
\mathrm{PS} \alpha=\frac{\mathrm{NP} q \alpha+\mathrm{NP} f \alpha+\mathrm{NP} b \alpha+\mathrm{NP} s \alpha+\mathrm{NPp} \alpha+\mathrm{NP} m \alpha+\mathrm{NP} l \alpha+\mathrm{NP} i \alpha}{8}
$$

Para calcular el índice sintético (IN) por cada estudiante a se consideró entonces,

$$
\mathrm{IN}^{\alpha}=\mathrm{PPN}^{\alpha-} \operatorname{DSP}^{\alpha / 2} \text {. }
$$

Por último, se procedió a hacer el filtrado de los casos extremos. Dada la distribución normal de los datos, aquellos estudiantes que exhibían desviaciones estándar con valores de 2,5 por encima de la media de la variable edad juliana se eliminaron. De igual forma, se depuró la base de datos consolidada, al eliminar las filas con información faltante. Como resultado, se llegó a una base de datos final depurada con un total de 25907 registros de estudiantes, de los cuales 18485 provienen de universidades y 7422 de instituciones técnicas. Esta base de datos final fue la utilizada para realizar los cálculos y análisis que se presentan a continuación. Para tal efecto, se llevaron a cabo en Excel tablas de contingencia y la prueba de Chi-Cuadrado con un nivel alfa de significancia del 1\%. Finalmente, se realizó la prueba $t$, de diferencias de dos medias, para comparar los resultados de la diferencia entre dos medias del índice sintético entre los estudiantes que siguieron sus estudios profesionales en universidades e instituciones tecnológicas, así como las pruebas Saber Pro de universidades públicas y privadas.

\section{Resultados}

Como era de esperarse, la distribución de ambas pruebas, el índice sintético de Saber 11 y el puntaje de Saber Pro es normal, según se aprecia en las figuras 1 y 2. Se colige que entre las dos la distribución de los resultados Saber Pro es de casi perfecta normalidad. 


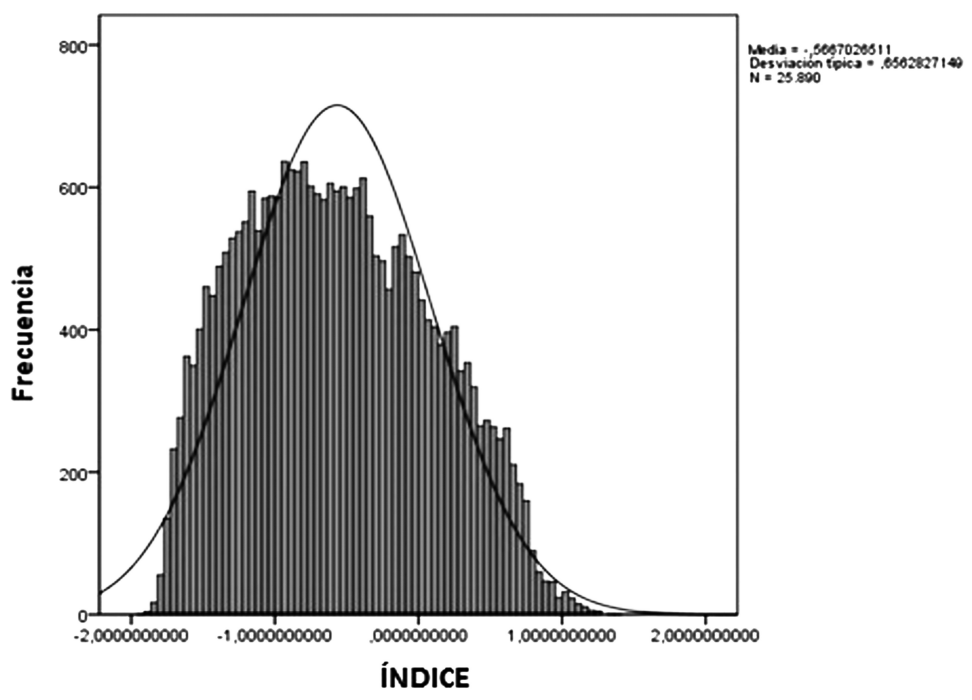

Figura 1. Distribución de frecuencia de los 25890 estudiantes seleccionados en el presente estudio que presentaron la prueba Saber 11.

Fuente: elaboración propia.

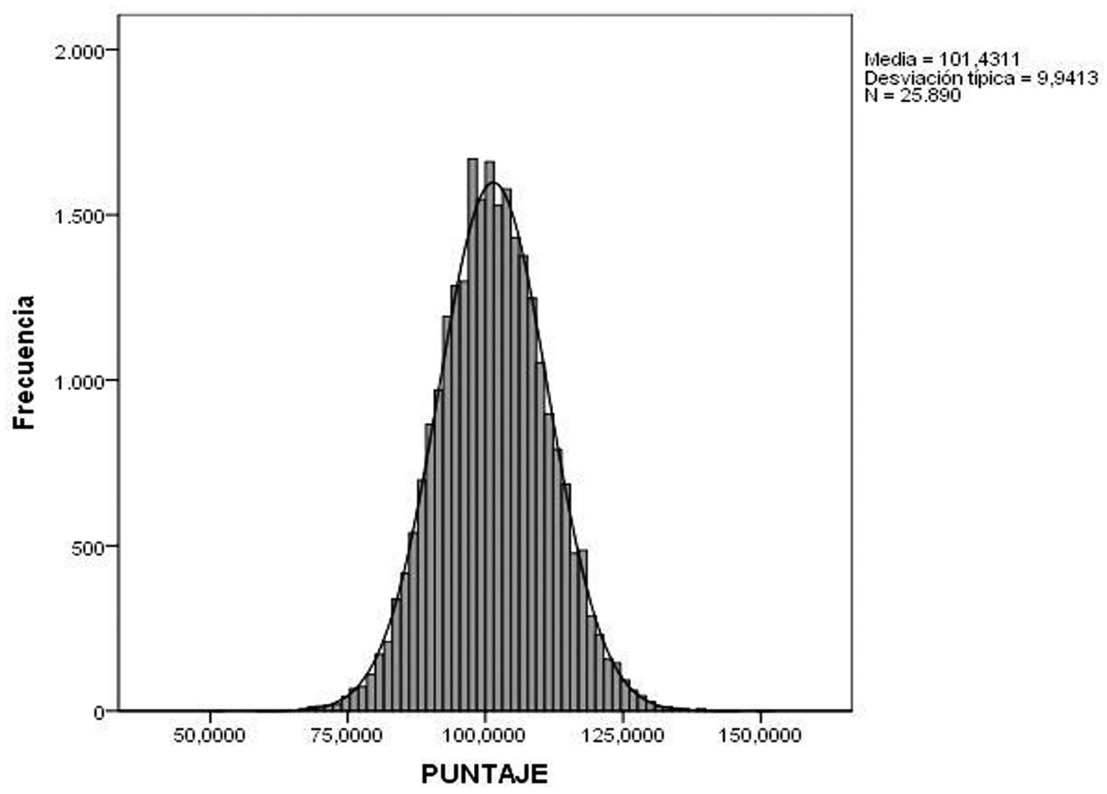

Figura 2. Distribución de frecuencias de la prueba Saber Pro 20092010, según los 25890 estudiantes seleccionados en el presente estudio. Fuente: elaboración propia. 
Hay aproximadamente dos mujeres por cada hombre que transitan satisfactoriamente del colegio a una institución de educación superior, de donde se gradúan. El calendario de los colegios se encuentra dominado por el A, con $86 \%$, seguido por el B, con $11 \%$, y por último el flexible, con 3\%. Un 37\% de los estudiantes de secundaria asiste a la jornada completa, mientras que el resto, $63 \%$, lo hace en jornada sabatina, mañana, tarde o noche. Con respecto al carácter de la institución secundaria, 61\% de los estudiantes asiste a un plantel académico, mientras que el resto, 39\%, a un plantel técnico, normalista u otro. El valor de la pensión de los estudiantes que transitaron satisfactoriamente de la secundaria a la universidad y que culminaron esta última se presenta en la tabla 1.

\section{Tabla 1}

Valor de la pensión mensual de la institución de educación media, en pesos, de los estudiantes que presentaron la prueba Saber 11 en el 2005-2006 y Saber Pro 2009-2010

\begin{tabular}{cc}
\hline Valor de la pensión mensual en pesos & Porcentaje \\
\hline No paga & 33 \\
$<33$,000 & 17 \\
33,000 a $<50,000$ & 3 \\
50, ooo a $<70,000$ & 4 \\
70,000 a $<100,000$ & 7 \\
100, o00 a $<150,000$ & 13 \\
150, o00 a $<250,000$ & 13 \\
$\geq 250,000$ & 10 \\
Total & 100 \\
\hline
\end{tabular}

Fuente: elaboración propia.

El estado civil de los estudiantes que presentaron las dos pruebas, en el momento de presentar la prueba Saber 11, era mayoritariamente soltero, con $96 \%$. El porcentaje restante, en partes iguales, divididos entre unión libre y casados (tabla 2). En el caso de los estudiantes que presentaron la prueba Saber Pro, los resultados son ligeramente diferentes, ya que surgen los divorciados. 
Tabla 2

Estado civil de los estudiantes que presentaron la prueba Saber 11 en el 2005-2006 y luego la prueba Saber Pro 2009-2010

\begin{tabular}{ccc}
\hline Estado civil & Saber 11 & Saber Pro \\
\hline Soltero/a & $96 \%$ & $94 \%$ \\
Unión libre & $2 \%$ & $2 \%$ \\
Casado/a & $2 \%$ & $2 \%$ \\
Separado/a & $0 \%$ & $1 \%$ \\
Viudo/a & $0 \%$ & $1 \%$ \\
Total & $100 \%$ & $100 \%$ \\
\hline
\end{tabular}

Fuente: elaboración propia.

A nivel profesional, los estudiantes que presentaron la prueba Saber Pro, en su mayoría, 68\%, asistieron a una universidad, mientras que el resto, 32\%, acudieron a una institución tecnológica.

La distribución porcentual por estrato de los estudiantes que tomaron la prueba Saber Pro en 2009-2010, y que además habían presentado la prueba Saber 11 en el 2005-2006, indica que la mayoría de estudiantes provienen de clase media. El porcentaje se reduce a medida que aumenta o se reduce el estrato (tabla 3).

Tabla 3

Distribución porcentual por estrato de los estudiantes que tomaron la prueba Saber Pro 2009 y 2010 y que habían presentado la prueba Saber 112005 y 2006.

\begin{tabular}{cc}
\hline Estrato & Porcentaje \\
\hline 1 & 8 \\
2 & 28 \\
3 & 37 \\
4 & 16 \\
5 & 7 \\
6 & 4 \\
Total & 100 \\
\hline
\end{tabular}

Fuente: elaboración propia. 
La media de la edad de presentación de la prueba Saber 11 es de 16,88 años o aproximadamente 16 años y 11 meses, con un rango de 12 años para unas edades entre 14 y 26 años. Para el caso de Saber Pro, la media de la edad es de 21,61 años o aproximadamente 21 años y 8 meses, con una edad mínima de 19 años y máxima de 30 años y 4 meses. Lo anterior arroja un rango de 11 años y 4 meses. El tiempo promedio que toma un estudiante entre ambas pruebas fue de 4,73 años o aproximadamente 4 años y 9 meses (tabla 4).

Tabla 4

Estadísticas descriptivas de la edad de los mismos estudiantes en la presentación de Saber 11 en 2005-2006 y Saber Pro 2009-2010.

\begin{tabular}{ccccc}
\hline Variable y & \multicolumn{4}{c}{ Estadístico } \\
\cline { 2 - 5 } Prueba & Media & Mínimo & Máximo & Rango \\
\hline Edad Saber 11 en años & 16.875 & 14.000 & 26.123 & 12.123 \\
Edad Saber Pro en años & 21.608 & 19.003 & 30.322 & 11.319 \\
\hline
\end{tabular}

Fuente: elaboración propia.

Como era de esperarse, los resultados de los índices sintéticos de Saber 11 son mayores para aquellos estudiantes que luego de la educación media siguieron en una universidad, en comparación con una institución técnica. Esta diferencia es estadísticamente significativa (tabla 5). De otro lado, los resultados de las pruebas son más dispersos en las universidades en comparación con las instituciones tecnológicas.

\section{Tabla 5}

Resultados del índice sintético de Saber 11 según carácter de la institución de educación superior -universidad, tecnológica y ambaspara los mismos estudiantes en la presentación de Saber 11 en 20052006 y Saber Pro 2009-2010

\begin{tabular}{cccc}
\hline & \multicolumn{2}{c}{ Estadístico } \\
Carácter de la Institución ${ }^{* *}$ & Media & Mediana & Desviación Estándar \\
\hline Universidades & -0.480 & -0.489 & 0.663 \\
Instituciones Tecnológicas & -0.783 & -0.851 & 0.583 \\
Ambas & -0.567 & -0.607 & 0.656 \\
\hline
\end{tabular}

Nota: **Prueba $t$ de diferencia entre dos medias entre universidades e instituciones tecnológicas p<0,01. Fuente: elaboración propia. 
Seis de cada diez estudiantes acudieron a un colegio o universidad del sector privado (tabla 6). Un poco más de la mitad de los estudiantes que culminaron la secundaria en un colegio oficial tienden a continuar sus estudios superiores en una universidad pública. Por el contrario, aproximadamente solo uno de cada cuatro egresados de colegios privados continúa sus estudios superiores en una entidad pública. Ambas relaciones anteriores son estadísticamente significativas (tabla 6).

\section{Tabla 6}

Tabla de contingencia de la naturaleza oficial o privada del colegio vs. naturaleza de la universidad - no incluye instituciones técnicas-, según los resultados de los estudiantes que presentaron las pruebas Saber 11 en 2005-2006 y Saber Pro 2009-2010

\begin{tabular}{|c|c|c|c|c|}
\hline & & \multicolumn{3}{|c|}{ Universidad } \\
\hline & & Privada & Pública & Total \\
\hline \multirow[t]{2}{*}{ Colegio } & Público & $\begin{array}{c}18.0 \% \\
(3,327) \\
\mathbf{4 5 , 7 \%}\end{array}$ & $\begin{array}{c}21.5 \% \\
(3,956) \\
54,3 \%\end{array}$ & $\begin{array}{c}39.4 \% \\
(7,283) \\
\mathbf{1 0 0 , 0 \%}\end{array}$ \\
\hline & Privado & $\begin{array}{c}43.8 \% \\
(8,096) \\
\mathbf{7 2 , 3 \%} \\
\end{array}$ & $\begin{array}{c}16.7 \% \\
(3,106) \\
\mathbf{2 7}, 7 \% \\
\end{array}$ & $\begin{array}{c}60.6 \% \\
(11,202) \\
100,0 \% \\
\end{array}$ \\
\hline \multicolumn{2}{|c|}{ Total } & $\begin{array}{c}61.8 \% \\
(11,423)\end{array}$ & $\begin{array}{c}38.2 \% \\
(7,072)\end{array}$ & $\begin{array}{c}100,0 \% \\
(18,485)\end{array}$ \\
\hline
\end{tabular}

Nota: Prueba de Chi-cuadrado de Pearson 1346, p<0,01 un grado de libertad. El número de estudiantes aparece en paréntesis y en negrilla los porcentajes de la fila. Fuente: elaboración propia.

Hay diferencias estadísticamente significativas por género en el índice sintético de Saber 11. Aproximadamente una de cada tres mujeres presentan puntajes bajos en comparación con uno de cada cuatro de los hombres (tabla 7). Además, considerando que hay aproximadamente dos mujeres por hombre en este estudio, se colige que, en términos relativos, más hombres logran puntajes altos que mujeres. Esto es, casi uno de cada cinco hombres, 19,8\%, mientras que aproximadamente una en siete de las mujeres logran los puntajes más elevados, $13,7 \%$. La relación es inversa en el caso 
de los bajos puntajes. Un mayor porcentaje de mujeres pertenece a esa categoría en comparación a los hombres, más de una en tres mujeres, mientras que un poco más de uno en cuatro, en el caso de los hombres.

Tabla 7

Tabla de contingencia entre género e índice sintético de Saber 11 para 2005-2006 y que culminaron estudios universitarios en 2009-2010

\begin{tabular}{|c|c|c|c|c|c|}
\hline & \multicolumn{5}{|c|}{ Puntaje índice sintético Saber 11} \\
\hline & & Bajo & Medio & Alto & Total \\
\hline \multirow[b]{3}{*}{ Género } & Femenino & $\begin{array}{c}23,2 \% \\
(4,289)\end{array}$ & $\begin{array}{c}33,8 \% \\
(6,248)\end{array}$ & $\begin{array}{c}9,1 \% \\
(1,682)\end{array}$ & $\begin{array}{c}66,2 \% \\
(12,237)\end{array}$ \\
\hline & & $\mathbf{3 5}, \mathbf{5 \%}$ & $51,0 \%$ & $13,5 \%$ & $100,0 \%$ \\
\hline & Masculino & $\begin{array}{c}9,2 \% \\
(1,701) \\
\mathbf{2 7 , 2 \%} \\
\end{array}$ & $\begin{array}{l}18,0 \% \\
(3,327) \\
\mathbf{5 3 , 2 \%} \\
\end{array}$ & $\begin{array}{c}6,7 \% \\
(1,238) \\
\mathbf{1 9 , 6 \%} \\
\end{array}$ & $\begin{array}{c}33,8 \% \\
(6,248) \\
\mathbf{1 0 0 , 0 \%} \\
\end{array}$ \\
\hline \multicolumn{2}{|c|}{ Total } & $\begin{array}{c}32,4 \% \\
(5,990) \\
\end{array}$ & $\begin{array}{c}51,8 \% \\
(9,575) \\
\end{array}$ & $\begin{array}{c}15,8 \\
(2,920) \\
\end{array}$ & $\begin{array}{c}100,0 \% \\
(18,485)\end{array}$ \\
\hline
\end{tabular}

Nota: Prueba de Chi-cuadrado de Pearson 180, p<0,01 dos grados de libertad. El porcentaje superior es sobre el total, el número de estudiantes aparece en paréntesis y el porcentaje de la fila en negrilla. Fuente: elaboración propia.

Hay una relación estadísticamente significativa entre los resultados de la prueba Saber 11 y Saber Pro. No obstante, cabe destacar que la gran mayoría de los estudiantes con bajos índices en Saber 11 lograron ubicarse en puntajes medios en Saber Pro e incluso algunos llegaron a altos puntajes (tabla 8). No es de extrañar que los resultados de los estudiantes con bajos índices, 7,7\% en Saber 11, continúan siendo así en la prueba Saber Pro, al terminar la universidad. Paralelamente, los estudiantes con altos índices en Saber 11 no migraron a bajos puntajes en Saber Pro, y aproximadamente uno de cada cinco estudiantes con altos puntajes en el índice sintético Saber 11 obtuvieron altos puntajes en la prueba Saber Pro. 
Tabla 8

Tabla de contingencia entre el índice sintético de Saber 11 para 2005-2006 y el puntaje de Saber Pro 2009-201O

\begin{tabular}{|c|c|c|c|c|c|}
\hline \multicolumn{6}{|c|}{ Puntaje Saber Pro } \\
\hline & & Bajo & Medio & Alto & Total \\
\hline \multirow{3}{*}{$\begin{array}{c}\text { Índice Sintético } \\
\text { Saber } 11\end{array}$} & Bajo & $\begin{array}{l}2,5 \% \\
(462) \\
7,7 \% \\
\end{array}$ & $\begin{array}{c}29,5 \% \\
(5,453) \\
\mathbf{9 1 , 1 \%} \\
\end{array}$ & $\begin{array}{l}0,4 \% \\
(239) \\
\mathbf{1 , 2 \%} \\
\end{array}$ & $\begin{array}{c}32,4 \% \\
(5,989) \\
\mathbf{1 0 0 , 0 \%} \\
\end{array}$ \\
\hline & Medio & $\begin{array}{l}0,8 \% \\
(148) \\
\mathbf{1 , 6 \%} \\
\end{array}$ & $\begin{array}{c}47,3 \% \\
(8,743) \\
\mathbf{9 1 , 3 \%} \\
\end{array}$ & $\begin{array}{l}3,7 \% \\
(684) \\
7,1 \% \\
\end{array}$ & $\begin{array}{c}51,8 \% \\
(9,575) \\
\mathbf{1 0 0 , 0 \%}\end{array}$ \\
\hline & Alto & $\begin{array}{l}0,0 \% \\
(0)\end{array}$ & $\begin{array}{c}12,9 \% \\
(2,385) \\
\mathbf{8 1}, 6 \% \\
\end{array}$ & $\begin{array}{r}2,9 \% \\
(536) \\
\mathbf{1 8 , 4 \%} \\
\end{array}$ & $\begin{array}{c}15,8 \% \\
(2,921) \\
\mathbf{1 0 0 , 0 \%} \\
\end{array}$ \\
\hline Total & & $\begin{array}{l}3,3 \% \\
(610)\end{array}$ & $\begin{array}{c}89,7 \% \\
(16,581)\end{array}$ & $\begin{array}{c}7,0 \% \\
(1,294)\end{array}$ & $\begin{array}{c}100,0 \% \\
(18,485)\end{array}$ \\
\hline
\end{tabular}

Nota: Prueba de Chi-cuadrado de Pearson 1395, p<0,01 cuatro grados de libertad. En paréntesis aparece el número de estudiantes y en negrilla el porcentaje de la fila. Fuente: elaboración propia.

La tabla de contingencia entre el carácter público o privado de la universidad y los puntajes de la prueba Saber Pro indican una relación estadísticamente significativa (tabla 9). De un lado, es evidente la mayor participación de estudiantes en instituciones privadas. No obstante, los estudiantes de estas tienen una mayor participación en los bajos puntajes y menor en los altos, en comparación con los egresados de las universidades públicas.

Tabla 9

Tabla de contingencia entre el carácter público o privado de la universidad y el puntaje Saber Pro 2009-2010 de aquellos estudiantes que presentaron la prueba Saber 11 en 2005-2006

\begin{tabular}{|c|c|c|c|c|c|}
\hline \multicolumn{6}{|c|}{ Puntaje Saber Pro } \\
\hline & & Bajo & Medio & Alto & Total \\
\hline \multirow[b]{2}{*}{$\begin{array}{l}\text { Carácter de la } \\
\text { universidad }\end{array}$} & Privada & $\begin{array}{l}2,2 \% \\
(407) \\
\mathbf{3 , 6 \%}\end{array}$ & $\begin{array}{c}56,1 \% \\
(10,370) \\
\mathbf{9 0 , 1 \%}\end{array}$ & $\begin{array}{l}3,4 \% \\
(629) \\
\mathbf{6 , 3 \%}\end{array}$ & $\begin{array}{c}61,8 \% \\
(11,406) \\
\mathbf{1 0 0 , 0 \%}\end{array}$ \\
\hline & Oficial & $\begin{array}{r}1,1 \% \\
(203) \\
\mathbf{2 , 9 \%}\end{array}$ & $\begin{array}{l}33,6 \% \\
(6,211) \\
87,7 \%\end{array}$ & $\begin{array}{l}3,6 \% \\
(666) \\
\mathbf{9 , 4 \%}\end{array}$ & $\begin{array}{c}38,2 \% \\
(7,079) \\
\mathbf{1 0 0 , 0 \%}\end{array}$ \\
\hline Total & & $\begin{array}{c}3,3 \% \\
(610)\end{array}$ & $\begin{array}{c}89,7 \% \\
(16,581)\end{array}$ & $\begin{array}{c}7,0 \% \\
(1,295)\end{array}$ & $\begin{array}{l}100,0 \% \\
(18,485)\end{array}$ \\
\hline
\end{tabular}


Nota: Prueba de Chi-cuadrado de Pearson 101, p<0,01 dos grados de libertad. En paréntesis aparece el número de estudiantes y en negrilla el porcentaje de la fila. Fuente: elaboración propia.

Una comparación entre los dos grupos de estudiantes, egresados de las universidades públicas y privadas, revela que los primeros presentan más altos puntajes en la prueba Saber Pro, siendo esta diferencia estadísticamente significativa. Además hay una mayor dispersión entre los puntajes de egresados de universidades oficiales que entre los egresados de las privadas (tabla 10).

\section{Tabla 10}

Estadísticas descriptivas de los puntajes de las pruebas Saber Pro 2009-2010 de estudiantes en universidades privadas y públicas y que presentaron la prueba Saber 11 en 2005-2006.

\begin{tabular}{ccc}
\hline & \multicolumn{2}{c}{ Universidad } \\
\hline Media** $^{*}$ & Privada & Pública \\
Mediana & 101,15 & 103,35 \\
Desviación Estándar & 101,10 & 103,36 \\
\hline
\end{tabular}

Nota: ${ }^{* *}$ Prueba $t$ de diferencia entre dos medias $\mathrm{p}<0,01$. Fuente: elaboración propia.

\section{Discusión}

Reiteramos que los resultados obtenidos son diferentes a los logrados si se estudiara separadamente el grupo Saber 11 y Saber Pro, tal y como lo hicieron Celis, Jiménez y Jaramillo (2012). En el presente trabajo se abordan los mismos estudiantes que presentaron ambas pruebas con cuatro o cinco años de diferencia. Colombia es el único país del mundo, a nuestro entender, que permite hacer dicho seguimiento; es decir, del mismo estudiante que exitosamente terminó la educación secundaria y la superior. No obstante, esta aproximación tiene sus fortalezas y debilidades. En primer lugar, deja por fuera a los estudiantes que no ingresaron a la universidad y a aquellos que, habiendo ingresado, no la terminaron en el periodo de tiempo que se consideró en este estudio. Dicha situación se exacerba al haber hecho el corte a los cinco años, en aras de la comparación de las pruebas, pues algunos estudiantes pueden demorarse seis o 
más años en terminar sus estudios superiores. No obstante, el hecho de poder seguir a los mismos estudiantes - a los exitosos, pues terminaron su educación secundaria y superior- ligando las dos pruebas Saber 11 y Saber Pro, arroja información muy valiosa que no sería posible obtener al estudiarlos separados. En otras palabras, los universos de cada prueba, Saber 11 y Saber Pro, se contaminarían por los estudiantes que tan solo terminaron la educación media y no continuaron a la educación superior o desertaron de esta, o que se tomaron más del tiempo aquí estipulado para terminar la educación superior.

El haber encontrado una distribución normal en las pruebas es un voto de confianza para el análisis de los resultados. Es sorprendente encontrar que el rango de edad es superior en los estudiantes de la prueba Saber 11 por casi 10 meses, en comparación con el rango de la prueba Saber Pro, a pesar de haber acotado la primera con un límite inferior de 14 años. Asimismo, resulta sorprendente el hallazgo de que, a tan temprana edad, un $1 \%$ de los estudiantes al culminar los estudios superiores se declaren viudos.

Dos de cada tres personas que terminan la educación superior son mujeres y lo hacen en una universidad; el resto, en una institución técnica. Como era de esperarse, los estudiantes con más altos índices sintéticos de Saber 11 continúan su educación superior en una universidad, en comparación con una institución técnica. También, los resultados de la tabla de contingencia entre el índice sintético de Saber 11 y el puntaje de Saber Pro sugieren que hay una relación estadísticamente significativa entre ambas pruebas. En otras palabras, los buenos estudiantes en la secundaria continúan siéndolo en la educación superior. Este desenlace se encuentra en sintonía con otros trabajos que usan la base de datos Saber (Melo, Ramos \& Hernández, 2014; Ramírez \& Teichler, 2014).

La tabla de contingencia entre la naturaleza del colegio y universidad sugiere una relación por medio dela cual las instituciones privadas de secundaria y superior captan más estudiantes que las entidades públicas. En efecto, seis de cada diez estudiantes que culminaron sus estudios profesionales provienen de un colegio y universidad del sector privado. No obstante, al considerar la calidad, medida en términos relativos de bajos y altos puntajes de la prueba Saber Pro, así como la media de los puntajes, se observa claramente 
la superioridad del sector oficial. Un estudiante de una universidad privada tiene el doble de probabilidad de presentar una prueba deficiente en comparación con los estudiantes de las universidades del sector oficial. De otro lado, un estudiante del sector oficial tiene $50 \%$ mayor probabilidad de presentar un alto puntaje en esta prueba en comparación con un egresado del sector privado.

Respecto a género, dos de cada tres personas que terminan la educación superior son mujeres y lo hacen en una universidad; el resto, en una institución técnica. Este estudio evidencia un mejor desempeño de los hombres en la prueba Saber 11. De igual forma, hay en la literatura académica colombiana más evidencia que soporta este hecho. Por ejemplo, la diferencia en matemáticas a favor de los hombres fue evidenciada por Caro y Casas (2013), la cual es significativa en las zonas urbanas y no significativa en las zonas rurales. Los mismos autores no encuentran una diferencia significativa en el caso de lenguaje. Guhl (2005), por su parte, encuentra más altos puntajes en los hombres que en las mujeres que presentaron examen de admisión a la Universidad Nacional de Colombia, incluso por carrera y edad.

Según los análisis de este estudio, al analizar por primera vez la vinculación entre las pruebas Saber 11 y Saber Pro, hay importantes implicaciones de política educativa. El Estado debe garantizar una educación de calidad en instituciones privadas de secundaria, que son la semilla para lograr buenos resultados en la educación superior, ya que el desempeño en calidad sugiere que estas se encuentran por debajo en calidad del sector oficial, tanto en la secundaria como en la universidad.

\section{Referencias}

Alonso, E., Rebolloso, E., Pozo, C., \& Fernández, B. (1999). Evaluación de la calidad en la educación superior. Papeles del Psicólogo, 74. Recuperado de https://dialnet.unirioja.es/servlet/articulo? codigo $=890897$

Ardila, M. (2011). Calidad de la educación superior en Colombia, ¿̇problema de compromiso colectivo?. Revista Educación y Desarrollo Social, $5(2), 44-55$.

Banco Mundial. (2009). La calidad de la educación en Colombia: un análisis y algunas opciones para un programa de política. Bogotá: Banco Mundial. 
Barrera-Osorio, F., Maldonado, D., \& Rodríguez, C. (2012). Calidad de la Educación Básica y Media en Colombia: Diagnóstico y Propuestas. Bogotá: Centro de Estudios sobre Desarrollo Económico, Facultad de Economía, Universidad de los Andes.

Burgess, S. \& Briggs, A. (2010). School Assignment, School Choice and Social Mobility. Economics of Education Review, 29(4), 639-649. doi: 10.1016/j.econedurev.2009.10.011

Camacho, A., Messina, J., \& Uribe, J. (2016). The Expansion of Higher Education in Colombia: Bad Students or Bad Programs? Washington D. C.: Interamerican Development Bank, Department of Research and Chief Economist. Recuperado de https://publications.iadb.org/ en/publication/12449/expansion-higher-education-colombia-badstudents-or-bad-programs

Caro, B. \& Casas. A. (2013). Análisis de las diferencias de género en el desempeño de estudiantes colombianos en matemáticas y lenguaje. Bogotá: Icfes.

Carter, D. \& O’Neill, M. (Eds.). (1995). International perspectives on educational reform and policy implementation. Washington, D. C.: Falmer Press.

Castro, M., Ruiz, J., \& Guzmán, F. (2017). Cruce de las pruebas nacionales Saber 11 y Pro en Antioquia, Colombia: una aproximación desde la regresión geográficamente ponderada (GWR). Revista Colombiana de Educación (en imprenta).

Celis, M., Jiménez, O., \& Jaramillo, J. (2012). ¿Cuál es la brecha de la calidad educativa en Colombia en la educación media y en la superior?. En Icfes Estudios sobre la calidad de la educación en (pp. 67-98).

Charniak, E. (1972). Toward a model of children's story comprehension (Ph. D. Dissertation, Massachusetts Institute of Technology, Cambridge, MA).

Chumacero, R., Gómez, D., \& Paredes, R. (2011). I Would Walk 500 Miles (if it paid). Economics of Education Review, 30(5), 1103-1114. doi: 10.1016/j.econedurev.2011.05.015

Faguet, J. \& Sánchez, F. (2008). Decentralization’s Effects on Educational Outcomes in Bolivia and Colombia, World Development, 36, 12941316. doi: 10.1016/j.worlddev.2007.06.021 
Ferrer, J. \& Arregui, P. (2003). Las pruebas internacionales de aprendizaje en América Latina y su impacto en la calidad de la educación: Criterios para guiar futuras aplicaciones. Programa de Promoción de la Reforma Educativa en América Latina y el Caribe. Grupo de Trabajo sobre Estándares y Evaluación. Recuperado de http://www.grade.org. pe/gtee-preal/docr/ExperLatino.pdf

Ferrer, J., Valverde, G., \& Esquivel, J. (1999). Aspectos del currículum prescrito en América Latina: Revisión de tendencias contemporáneas en currículum, indicadores de logro, estándares y otros instrumentos. Programa de Promoción de la Reforma Educativa en América Latina y el Caribe. Grupo de Trabajo sobre Estándares y Evaluación. Recuperado de https://issuu.com/guerrerortiz/docs/ferreraspectosdelcurriculumal/1

Gaviria A. \& Barrientos J. (2001). Determinantes de la calidad de la educación en Colombia. Archivos de Economía, 159. Recuperado de https:// colaboracion.dnp.gov.co/CDT/Estudios\%20Econmicos/159.pdf

Gómez, S. (2016). Educational achievement at schools: Assessing the effect of the civil conflict using a pseudo-panel of schools. International Journal of Educational Development, 49, 91-106. http://dx.doi. org/10.1016/j.jjedudev.2016.02.004

Guhl, A. (2005). ¿Qué tan nacional es la Universidad Nacional de Colombia?. Serie Documentos de Trabajo, (7). Bogotá: Universidad Nacional de Colombia.

Icfes. (2008). Resolución $N^{\circ} 489$ de octubre 20 de 2008. Por la cual se adoptan las metodologías para seleccionar los mejores estudiantes de la Prueba de Estado Aplicada por el ICFES y para la clasificación de las Instituciones Educativas.

Linn, R. \& Herman J. (1997). La evaluación impulsada por estándares: problemas técnicos y políticos en la medición del progreso de la escuela y los estudiantes. Los Ángeles: Universidad de California.

Loaiza, O. \& Hincapié, D. (2016). Un estudio de las brechas municipales en calidad educativa en Colombia: 2000-2012. Ensayos sobre Política Económica, 34, 3-20. http://dx.doi.org/10.1016/j.espe.2016.01.001 0120-4483

López, N. (2007). Acerca de la problemática de la evaluación de la calidad de la educación en Colombia. Revista Latinoamericana de Estudios Educativos, 3, 9-28. Recuperado de http://www.redalyc.org/articulo. 
oa? id=134112600002ER

Melguizo, T., Sánchez, F., \& Velasco, T. (2016). Credit for Low-Income Students and Access to and Academic Performance in Higher Education in Colombia: A Regression Discontinuity Approach. World Development, 8o, 61-77. doi: 10.1016/j.worlddev.2015.11.018

Melo, L., Ramos, J., \& Hernández, P. (2014). La Educación Superior en Colombia: Situación Actual y Análisis de Eficiencia. Borradores de Economía, 808. Recuperado de http://www.scielo.org.co/pdf/dys/ n78/n78ao3.pdf

Mina, A. (2004). Factores asociados al logro educativo a nivel municipal. Bogotá: Universidad de los Andes. Recuperado de https:// economia.uniandes.edu.co/component/booklibrary/478/view/46/ Documentos\%20CEDE/467/factores-asociados-al-logro-educativo-anivel-municipal

Montoya, F. (2010). Evolución de los resultados de las pruebas Icfes y Saber en las áreas de matemática y lenguaje en Risaralda. Revista Académica e Institucional, 88, 97-111.

OECD \& World Bank. (2013). Reviews of National Policies for Education: Tertiary Education in Colombia 2012. Recuperado de https:// www.oecd-ilibrary.org/education/reviews-of-national-policies-foreducation-tertiary-education-in-colombia-2012_9789264180697-en

Orden de la, A. (2009). Evaluación y calidad: análisis de un modelo. Estudios sobre Educación, 16, 17-36.

Orozco, L. (2008). La cobertura en la educación superior. Mito y realidad. Recuperado de Agregar:https://razonpublica.com/ index.php/econom-y-sociedad-temas-29/171-la-cobertura-en-laeducaciuperior-mito-y-realidad.html

Pinto, L. (1999). Currículo por competencias: Necesidad de una nueva escuela. Tarea, Revista de Educación y Cultura, 43, 10-17. Recuperado de https://www.worldcat.org/title/curriculo-por-competenciasnecesidad-de-una-nueva-escuela/oclc/820215307

Piron, L. \& O'Neill, T. (2005). Integrating Human Rights into Development: A Synthesis of Donor Approaches and Experiences. London: ODI.

Ramírez, C. \& Teichler, U. (2014). Factores socioeconómicos y educativos asociados con el desempeño académico, según nivel de formación 
y género de los estudiantes que presentaron la prueba SABER $P R O$ 2009. Bogotá: Icfes, Estudios sobre calidad de la educación en Colombia.

Rodríguez, C., Sánchez, F., \& Armenta, A. (2010). Do Interventions at School Level Improve Educational Outcomes? Evidence from a Rural Program in Colombia. World Development, 38(3), 415-428. doi: 10.1016/j. worlddev.2009.10.002

Saavedra, J. (2012). Resource constraints and educational attainment in developing countries: Colombia 1945-2005. Journal of Development Economics, 99, 80-91. doi: 10.1016/j.jdeveco.2011.09.006

Tikly, L. \& Barrett, A. (2011). Social Justice, Capabilities and the Quality of Education in Low Income Countries. International Journal of Educational Development, 31(1), 3-14. doi: 10.1016/j. ijedudev.2010.06.001

Tünnermann, C. (2008). La calidad de la educación superior y su acreditación: la experiencia centroamericana. Revista da Avaliação da Educação Superior, 13(2), 313-336. Recuperado de: http://www. redalyc.org/articulo.oa?id=219114873005ER

Unicef. (2010). Defining Quality in Education. New York, NY: Education Section Programme Division.

Valverde, G. (1997). Evaluación y estándares de currículo en una era de reforma educativa. En B. Álvarez \& M. Ruiz-Casares (Eds.), Evaluación y Reforma Educativa: Opciones de política (pp. 57-88). Washington D. C.: U.S. Agency for International Development.

Winters, J. (2011). Human Capital, Higher Education Institutions, and Quality of Life. Munich Personal RePEc Archive. doi: 10.1016/j. regsciurbeco.2011.03.001 